\title{
Largest vaccine trial in Africa shows protection against leprosy but not tuberculosis
}

Results of the largest vaccine trial ever carried out in Africa are reported in the Lancet, 1996; 348: 17-24.

More than 120,000 people in Karonga District, Northern Malawi were followed up for 5-9 years after receiving one or two BCG vaccines, or BCG plus killed leprosy bacilli. Results show that two doses of BCG reduced the incidence of leprosy by $75 \%$, but did not protect against tuberculosis. The protection against leprosy was particularly evident among younger individuals. There was no evidence that the addition of killed leprosy bacilli contributed to protection against either disease.

The trial demonstrates that $\mathrm{BCG}$ - the most widely-used vaccines in the world - can have a considerable impact against leprosy, but none against tuberculosis, in the same population. The findings provide an important guide for leprosy control, and a key for research on new tuberculosis vaccines, by revealing disease-specific activity of BCG which differs within and between populations.

The trial commenced in 1986, in co-operation with the Government of Malawi and the people of the Karonga District. It was designed and directed by Professor Paul Fine of the London School of Hygiene and Tropical Medicine and funded by the British Leprosy Relief Association (LEPRA), other antileprosy agencies and the World Health Organization. It represents one of the largest and most detailed controlled clinical studies ever undertaken (see below for details).

$\begin{array}{llll}\text { Contacts: } & \text { Professor Paul Fine } & \text { (LSHTM) } & \text { Tel: } 01719272219 \\ & \text { Dr Jo Colston } & \text { (LEPRA) } & \text { Tel: } 01819593666 \text { (ext 2354) } \\ & \text { Mr Terry Vasey } & \text { (LEPRA) } & \text { Tel: } 01206562286\end{array}$

London School of Hygiene \& Tropical Medicine (LSHTM), Department of Epidemiology \& Population Sciences, Keppel Street, London WC1E 7HY

\section{Supporting statement}

Effective vaccines are urgently needed in the fight to control leprosy and tuberculosis, the most important mycobacterial diseases in the world. Leprosy is still widespread, but its elimination as a public health problem by the year 2000 is one of the major targets of the World Health Organization. Tuberculosis is now responsible for more deaths than is any other infectious disease, and has been declared a global emergency by the WHO. BCG vaccines are employed against both these diseases but are effective to a variable degree, and a major international research effort is now underway to develop and test new vaccines. 
Though repeat BCG vaccination has been policy in many countries for prevention of tuberculosis and leprosy, its effectiveness has never been evaluated. It has been proposed that adding antigens of the leprosy bacillus to BCG might increase its effectiveness against leprosy. A double-blind randomized controlled trial to evaluate both these procedures was carried out in a rural area in Malawi, where a single BCG delivered by the routine health services had previously been found to halve the incidence of leprosy but to provide no protection against tuberculosis.

The trial began in 1986. 121,000 people were randomized to receive either a single (Glaxo) BCG, two BCG vaccines, or a new experimental vaccine combining BCG plus killed leprosy bacilli. Results obtained after 5-9 years of follow-up revealed that a second dose of BCG vaccine again halved the incidence of leprosy indicating that a two-dose schedule could prevent $75 \%$ of cases of leprosy. The protection appeared greatest among younger individuals (less than 15 years of age at vaccination). On the other hand, the additional BCG still failed to provide any protection against tuberculosis, and there was no evidence that the addition of killed leprosy bacilli contributed to protection against either disease.

These results provide an important new perspective on the action of BCG, which is both the most widely used yet most controversial vaccine in the world. They have immediate implications both for disease control and for research priorities. The evidence for appreciable protection against leprosy but no protection against tuberculosis, in the same population, indicates that the poor performance of BCG vaccines against tuberculosis in many tropical areas is attributable to selective immunological response rather than to problems with handling or administration of the vaccines. The demonstration of this clear dichotomy in BCG's effect provides a key to the riddle of BCG's variable behaviour, which has obstructed efforts to develop improved mycobacterial vaccines.

The results further confirm that BCG vaccination is playing a major role in reducing leprosy incidence worldwide, and indicate that repeated BCG vaccination of high-risk individuals will be an appropriate strategy in populations where leprosy is common.

The BCG vaccine employed in this trial, and in the Malawi national programme, is identical to that used in routine $\mathrm{BCG}$ vaccination in the $\mathrm{UK}$, where it is known to impart consistently high (approximately 80\%) protection against tuberculosis. Comparison of the responses to this vaccine in the UK and in Malawi should help researchers to understand the differences in vaccine action in different populations and hence to design better vaccines against both leprosy and tuberculosis.

The Karonga Prevention Trial is the largest vaccine trial ever carried out in Africa, the first trial ever carried out of repeat BCG vaccination, and the only combined leprosy-tuberculosis vaccine trial in the world. The trial was designed and directed by Professor P. E. M. Fine and Dr J.M. Ponninghaus from the London School of Hygiene and Tropical Medicine, and was supported primarily by the British Leprosy Relief Association (LEPRA), with co-operation of the Malawiian Ministry of Health. Additional support came from the International Federation of Anti-leprosy Organisations (ILEP) and the UNDP/World Bank/World Health Organization Special Programme on Research and Training in Tropical Diseases. 


\section{Reference}

Karonga Prevention Trial. Randomized controlled trial of single BCG, repeated BCG, or combined BCG plus killed Mycobacterium leprae vaccine, for prevention of leprosy and tuberculosis in Malawi. Lancet 1996; 348: $17-24$.

LEPRA, The British Leprosy Relief Association, is a medical charity, founded in 1924. The current Director is Mr Terry Vasey. Dr Jo Colston is Head of the Medical Research Council's Mycobacterial Laboratory at the National Institute of Medical Research, and is Chairman of LEPRA's Medical Advisory Board. LEPRA's main objective is the eradication of leprosy, in pursuit of which it supports field control programmes and funds research aimed at combating the disease. Because of the association between tuberculosis and leprosy, LEPRA is also involved in research and control of tuberculosis. For further details please contact LEPRA, Fairfax House, Causton Road, Colchester CO1 1PU, UK. Tel: 44(0)1206 562286; fax: 44(0)1206 762151.

The London School of Hygiene \& Tropical Medicine is a post-graduate medical school of the University of London and the leading institution in Europe for public health and tropical medicine. For further details of this paper and of the work of the LSHTM, please contact Dr Barbara Judge, Project Officer 44(0)171-927-2464), London School of Hygiene \& Tropical Medicine, Keppel Street, London WC1E 7HT, UK. 